\title{
Screening for Syphilis in Pregnant Women in Resource-Limited Environment: Which Approach Is Effective? Case Report
}

\author{
Raphael Byamungu Mugondozi, Gaspard Makambo Mohilo, Maurice Masoda Nyamalyongo \\ Family Medicine and Primary Health Care Department of "Protestant University of Congo", Kinshasa, DR Congo \\ Email: byamunguraphael@gmail.com
}

How to cite this paper: Mugondozi, R.B., Mohilo, G.M. and Nyamalyongo, M.M. (2021) Screening for Syphilis in Pregnant Women in Resource-Limited Environment: Which Approach Is Effective? Case Report. Open Access Library Journal, 8: e7093. https://doi.org/10.4236/oalib.1107093

Received: December 18, 2020

Accepted: May 22, 2021

Published: May 25, 2021

Copyright $\odot 2021$ by author(s) and Open Access Library Inc.

This work is licensed under the Creative Commons Attribution International License (CC BY 4.0).

http://creativecommons.org/licenses/by/4.0/ (c) (i) Open Access

\begin{abstract}
Syphilis in a pregnant woman keeps threatening the life of both the woman and her fetus at any time of pregnancy. While the control of this disease seemed to be effective around the 2000s in developed countries, its prevalence in pregnant women has risen all over the world especially in countries with limited resources. Although syphilis control in pregnant women would be part of the health policy of almost all the countries of sub-Saharan Africa, this opportunity is unfortunately not capitalized to the maximum by pregnant women who, for the most part, do attend health facilities only during the last trimesters of pregnancy. The purpose of this case study is to evaluate effective methods in screening pregnant women for syphilis in the absence of an equipped laboratory, which is the situation in many low income countries.
\end{abstract}

\section{Subject Areas}

Epidemiology, Global Health, Gynecology \& Obstetrics, Infectious Diseases, Women's Health

\section{Keywords}

Screening for Syphilis, in Pregnant Women, Resource-Limited Environment

\section{Introduction}

Syphilis is a sexually transmitted disease caused by bacteria "Treponema pallidum". It can progress to systemic disease at any time if left untreated [1]. This disease has disastrous effects when it occurs during pregnancy; leading to several complications for both the woman and the fetus. Among the main complications of congenital infection we can cite abortions, prematurity, stillborn, perinatal death, low birth weight, congenital anomalies, active syphilis in the newborn or 
the sequelae to long term [2] [3].

Although it is one of the easily preventable sexually transmitted diseases and the treatment of which is widely known, its prevalence in pregnant women has increased markedly in developing countries in recent decades [1] [3].

In 2013, WHO reported 1.9 million pregnant women infected with syphilis worldwide with $66.5 \%$ of adverse fetal outcomes if no treatment [3]. In some parts of the world, the seroprevalence of syphilis among pregnant women was estimated in 2019 at $4.4 \%$ [4].

In sub-Saharan Africa, the prevalence of syphilis among pregnant women is estimated at $2.7 \%$, accounting for nearly one million at risk pregnancies each year [5].

In DR Congo, in the report of the National AIDS Control Program (PNLS) of 2017 [6], the overall prevalence of syphilis was $2.12 \%$ among pregnant women attending the prenatal consultation, with rate variations depending on the province. This shows the potential risk within the general population with the need for a good health system organizing early management of syphilis in pregnant women in order to prevent its complications.

The control of syphilis is facilitated by the availability of inexpensive and sensitive diagnostic tests, and of effective and affordable treatment. This is one of the effective measures that healthcare workers can use to reduce syphilis in pregnant women and their impact on pregnancy and the morbidity and mortality associated with congenital syphilis [7]. Yet, doubts are still expressed about the effectiveness of the currently using syphilis tests [8].

This is what motivated us to present this case study on the efficacy of screening test for syphilis from the experience of a pregnant woman followed in the gynecological-obstetrics department at Heal Africa Hospital, Goma city in DR Congo.

\section{Case Presentation}

A 30-year-old woman, married and mother of one child. She consulted on April 13, 2020 complaining about genital bleeding after 3-month amenorrhea. She stated to have nausea, anorexia, dyspareunia and mictalgia as well.

In the background, gestation 3, parity 1, abortion 1. Date of last period: January 11,2020 . She had an abortion of the 2 nd pregnancy at five months 4 months ago and the cause was not reported to her. No previous Antenatal Care (ANC) since beginning of amenorrhea. Her husband and her child have no complain.

Physical examination of other systems was normal. But gynecological examination noted the presence of blood on the vaginal wall and on the exocervix. The cervix is long, closed, median, with tenderness.

The pelvic ultrasound concluded in a 6-week mono ovular intrauterine pregnancy, with decidual hematoma. This did not correlate with the gestational age (according to last period date).

Laboratory test result. blood group and rhesus: Brh+; urinary sediment: 15 
white cells/field (reference range: under 10 white cells/field), 90 epithelial cells/field; hemoglobin: $13.1 \mathrm{~g} / \mathrm{dl}$ (reference range: 12 - $16 \mathrm{~g} / \mathrm{dl}$ ), hematocrit: 40\% (reference range: $36 \%$ - 48\%), C-reactive protein (CRP) positive, Venereal Disease Research Laboratory (VDRL)/Syphilis test and Treponema Hemagglutination (TPHA) test results were positive. HbsAg, Toxoplasmosis, Rubella, Malaria tests were negative.

We concluded to an active syphilis complicated by a threatened abortion, and the treatment started with intramuscular benzanthine penicillin 2.4 million international units. We asked the patient to remain in a close monitoring of the pregnancy in the maternity ward, physical rest, and $180 \mathrm{mg}$ of spasfon in $500 \mathrm{ml}$ of physiological saline in IV line for 6 hours. Seven days later a second dose of bezantine penicillin and the follow-up of her spouse were done.

On April 18, 2020, the patient had no complain. We appreciated the good clinical outcome and decided patient to be discharged. And an appointment was set 7 days later for a check-up and follow-up of the pregnancy.

On May 2, 2020, the patient returned for a check-up. The obstetric ultrasound examination revealed a stopped pregnancy of 9 weeks.

We prepared the family for this bad news and the next step, was to evacuate uterine content one day later. She started with sublingual misoprostol $600 \mathrm{mi}-$ crograms; this dose was repeated 6 hours later, which allowed to evacuate the dead embryo successfully. Figure 1 shows the results of ultrasonic examination.

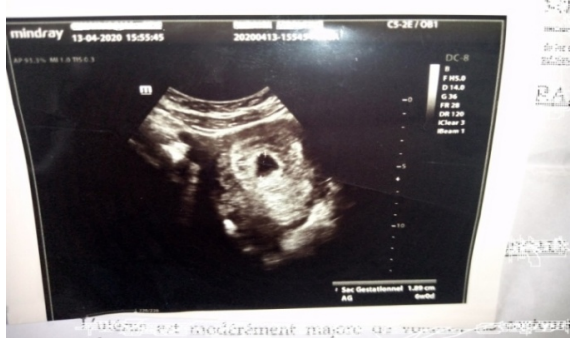

(a)

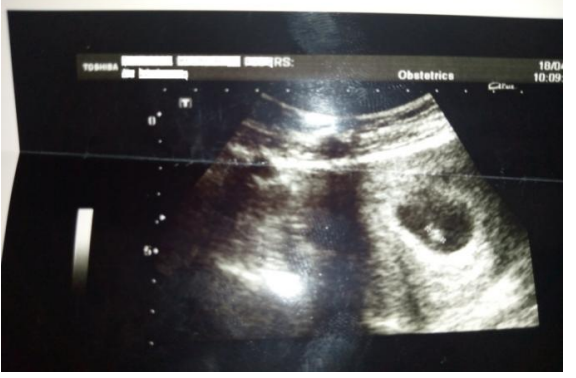

(b)

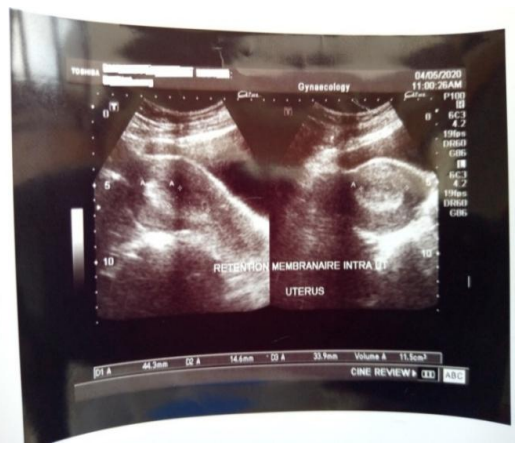

(c)

Figure 1. Results of ultrasonic examination. (a) Obstetrical ultrasound: amniotic sac on April 13, 2020; (b) Obstetrical ultrasound: a uni embryonic intrauterine pregnancy on April 18, 2020; (c) Pelvic ultrasound: intrauterine membrane retention on May 4, 2020 (two days after abortion). 


\section{Discussion}

A patient is considered to have syphilis when one or more tests are positive. These tests are: Treponema Hemagglutination (TPHA), Enzyme Immunoassay (EIA), Venereal Disease Research Laboratory (VDRL), Rapid Plasma Reagin (RPR), Enzyme Linked Immunosorbent Assay (ELISA) and Rapid Immunochromatographic test (RICT) [5], with or without the presence of symptoms and clinical signs due to this disease [8].

\subsection{Clinical Features and Developmental Stages of the Disease}

Syphilis is transmitted through direct person-to-person contact with an open syphilitic wound and through vaginal, anal or oral sex. The external genitalia, vagina, rectum or anus, and the lips and inside of the mouth are the main organs where syphilitic lesions usually occur. Additionally, syphilis can be transmitted vertically to the fetus from an infected pregnant woman during pregnancy or childbirth [9], and through blood transfusion [10].

In its course outside of treatment, the disease passes through different stages characterized by different symptoms and signs. These stages include primary, secondary, tertiary syphilis, early latent or late latent syphilis [11].

Adverse outcomes occur in 50\% - 80\% of pregnancies that survive beyond 12 weeks in infected women [10] [12]. The likelihood of fetal damage occurs in women with active syphilis infection, especially when it is insufficiently or untreated, acquired during the last five years preceding pregnancy [9]. The risk of congenital transmission begins around 14 weeks gestation [13].

As observed in our case study, according to the same authors, $25 \%$ of women with active syphilis may present late abortions and stillbirths, $11 \%$ of cases of full-term neonatal death, $13 \%$ of cases of prematurity or low birth weight, and $20 \%$ of the classic symptoms and clinical signs of congenital syphilis [9].

\subsection{Potential Opportunities to Control the Threat of Syphilis}

Despite screening and treatment methods that are widely known, available and affordable, syphilis remains among the pathologies constituting a major health challenge in pregnant women, particularly in sub-Saharan Africa [14]. Preventable congenital syphilis infections continue to occur because pregnant women, especially those who are poor or who live in rural areas without access to quality services, are often not screened according to national guidelines. Yet, early treatment is needed to minimize or eliminate mortality and morbidity from this disease [13].

To achieve the goals of eliminating mother-to-child transmission of syphilis as well as HIV, the WHO has been advocating since 2014 for guidance on criteria and validation processes. According to this policy, countries must meet the programmatic objectives of systematic syphilis screening (target $95 \%$ of all pregnant women) and treatment (target $95 \%$ of women who test positive and put on treatment). Adverse perinatal outcomes caused by maternal syphilis infection 
can be avoided with this health policy [12] [15].

According to the recommendation of the WHO, the first visit of ANC must take place within 12 weeks of the pregnancy and at least 4 visits are necessary at a specific interval for a pregnancy without medical particularities. This strategy would allow early diagnosis of pathologies in pregnant women including syphilis, and the early initiation of treatment for positive cases [14]. But for this to be effective, serological screening should be done at the first ANC visit as recommended, although few women observe this measurement. The ideal would be to carry out these serological tests twice during pregnancy: During the first trimester and early during the third, and this regardless of the prevalence of syphilis in the population [16].

\subsection{Laboratory Examinations}

The diagnosis of syphilis is complicated due to the extreme difficulty in growing Treponema pallidum in vitro [17]. As a result, lab technicians have been forced to focus on other methods of diagnosing syphilis [18]. Serological testing for syphilis, with the detection of non-treponemal antibodies or antibodies to Treponema pallidum at all stages of infection, remains the mainstay of diagnosis. These tests detect IgM and IgG antibodies [19]. Non-treponemal tests (NTT) are widely used to monitor the status of infection, while treponemal tests (TT) are mainly used to confirm the presence of treponemal infection [17] [18].

\subsubsection{Non-Treponemal Tests}

All non-treponemal tests detect both IgM and IgG antibodies, usually detectable as early as the 6th day after infection, with a sensitivity of around 75\% [18].

According to Phang Romeo CC et al. [18] NTT offer high sensitivity, but not for the initial stage of primary syphilis or the late period of the disease (tertiary syphilis), hence the limitations of these types of serological tests requiring a good interpretation of these results. In a study by Negash $\mathrm{M}$ et al. [10] consisting in evaluating the sensitivity, specificity, positive predictive value and negative predictive value by comparing the different serological tests, for the RPR these values were respectively $100 \%, 80.8 \%, 76 \%, 2 \%$ and $100 \%$. However, at the end of this study, following the possibility of finding a high rate of false positive results with the use of the RPR serological test, the authors concluded that the latter should not be used in isolation for diagnosis or for syphilis screening because these tests are not specific for Treponema pallidum [16]. Although cheaper and easier to use, it is better indicated for monitoring response to treatment for syphilis [8], because false positives result may occur [18]. This should limit the isolated use of non-treponemal tests [16].

\subsubsection{Treponemal Tests}

The sensitivity and specificity of TT vary depending on the type of test as well as the stage of infection with syphilis. In addition, although the subspecies of Treponema pallidum is the most common species in developed countries where 
these tests are generally made, there are other subspecies of Treponema pallidum which differ in their pathogenicity but are homologous in $95 \%$ by DNA-DNA hybridization and cannot be distinguished by these serological tests [18]. TT are more sensitive and specific than NTT. However, since antibodies continue to circulate for life after treatment, these tests cannot distinguish between an active infection and a previous infection [17].

Indeed, these tests detect either IgM or IgG antibodies, depending on the specific test kit. Most diagnostic laboratories use the TPHA, the "Treponema pallidum Particle Agglutination" (TPPA) and/or the "Fluorescent Treponemal Antibody Absorption" (FTA-ABS) test). Antibodies detected by these TT occur earlier than those detected by the NTT and remain detectable for life, even after successful treatment. But sensitivity and specificity of TPHA and TPPA are not optimal for primary syphilis [8] [18].

\subsection{Syphilis Diagnostic Algorithms}

Most patients infected with syphilis are either asymptomatic or with transient lesions, hence serological testing is often the preferred detection method. In order to avoid errors in the interpretation of diagnostic test results as much as possible, the evidence is that, unlike what is observed in several regions of the world where screening for syphilis is made of only one type serological testing, advocacy should be carried out with a view to introducing the diagnostic confirmation test into medical practice during antenatal care [16].

According to Rac MW et al. [20] there are 2 diagnostic algorithms of which each doctor should be aware of the type available in the structure where he practices.

First algorithm: The serological test consists of using one of the NTT such as the RPR or VDRL test. The positive result is then confirmed by use of a specific TT such as TPPA or TPHA or even FTA-ABS [11]. This is the traditional approach used for several years.

Second algorithm: It's just a reverse approach to the first one. In fact, over the past few years, due to the need for efficiency in mass screening as well as the need to respond to a large number of samples, many laboratories have changed their diagnostic approach and now proceed by use first a treponemal antibody test in which the blood sample is tested using the enzyme immunoassay (EIA) method. Positive samples are then tested with a quantitative NTT such as an RPR or VDRL test. If the test results do not match, a second TT test is used. TPPA is generally recommended as a confirmatory test. This is the algorithm currently approved and recommended by researchers [8].

On the other hand, according to Muhammad G et al. [18] in addition to two algorithms commonly used for the diagnosis of syphilis, a variant of the reverse sequence algorithm is recommended by the European Center for Disease Prevention and Control (ECDC). According to this variant, a reactive treponemal screening test is followed by a second different TT, without using the NTT. Note 
that these algorithms for the diagnosis of syphilis as detailed above are mainly used in regions and countries with a low prevalence of syphilis and good laboratory coverage, where we may not recognize the place and role of rapid tests in improving the diagnosis of syphilis [21].

However, in low- and middle-income countries, many health facilities that organize screening and treatment for syphilis during antenatal visits do not have the capacity to perform confirmatory diagnostic tests as required by these syphilis diagnostic algorithms. These tests are laboratory dependent and require trained personnel, good storage of reagents in the refrigerator, and constant electricity to run the devices [16]. Additionally, patients may not return for lab results, and specimens and results may get lost, leading to treatment delay or failure [16] [17].

To overcome this challenge, currently new tests involving cloning of Treponema pallidum antigens and an immunochromatographic technique constitute an alternative to rapid treponemal tests to be performed at the point-of-care. This is the immunochromatographic syphilis test (ICS). It lasts around $20 \mathrm{mi}-$ nutes and is generally considered quite sensitive and specific. In addition, the test format does not require the use of special equipment such as fluorescence microscopes or microplate readers, making it an ideal site-of-care test. This test has the advantage of allowing women with a positive result to be treated on-site at the same visit, thus avoiding the requirements for return for follow-up and the potential side effects associated with delayed treatment [17]. It may be more convenient and efficient than traditional diagnostic algorithms in antenatal care settings where prompt treatment is essential [12]. This test meets the criteria set by the Special Program for Research and Training in Tropical Diseases of the WHO. According to him, the ideal characteristics of the tests to be used in the point-of-care are as follows: they are affordable, sensitive, specific, simple, rapid and robust; and can reasonably be used as an initial screening test for syphilis [17].

\section{Conclusions}

The traditional diagnostic algorithm used in several regions, initially proceeding with non-treponemal serological tests has many limitations since these tests are false negative at the initial and late latent stages of the disease, which unfortunately rules out the use of treponemal serological tests, which are considered more effective.

Given the difficulties of access to laboratory equipment, trained personnel and difficult working conditions in countries with limited resources, the advocacy towards the use of ICS at point-of-care should be part of our duty in order to improve our service in such a setting.

\section{Conflicts of Interest}

The authors declare no conflicts of interest regarding the publication of this paper. 


\section{References}

[1] Stafford, I.A., Berra, A., Minard, C.G., Fontenot, V., Kopkin, R.H., Rodrigue, E., et al. (2019) Challenge in the Contemporary Management of Syphilis among Pregnant Women in New Orleans, LA. Infectious Diseases in Obstetrics and Gynecology, 2019, Article ID: 2613962. https://doi.org/10.1155/2019/2613962

[2] Assefa, A. (2014) A Three Year Retrospective Study on Seroprevalence of Syphilis among Pregnant Women at Gondar University Teaching, Ethiopia. African Health Sciences, 14, 119-124. https://doi.org/10.4314/ahs.v14i1.18

[3] Pedreira de Cerqueira, L.R., Monteiro, D.L.M., Taquette, S.R., Rodrigues, N.C.P., Trajano, A.J.B., Monteiro de Souza, F., et al. (2017) The Magnitude of Syphilis: From Prevalence to Vertical Transmission. Revista do Instituto de Medicina Tropical de São Paulo, 59, e78. https://doi.org/10.1590/s1678-9946201759078

[4] Benedetti, K.C.S.V., Ribeiro, A.D.D.C., Queiroz, J.H.F.S., Melo, A.B.D., Batista, R.B., Delgado, F.M., et al. (2019) High Prevalence of Syphilis and Inadequate Prenatal Care in Brazilian Pregnant Women: A Cross-Sectional Study. American Journal of Tropical Medicine and Hygiene, 101, 761-766.

https://doi.org/10.4269/ajtmh.18-0912

[5] Hussen, S. and Tadesse, B.T. (2019) Prevalence of Syphilis among Pregnant Women in Sub-Saharan Africa: A Systematic Review and Meta-Analysis. BioMed Research International, 2019, Article ID: 4562385. https://doi.org/10.1155/2019/4562385

[6] Programme National de Lutte contre le VIH/Sida et IST. Rapport annuel 2017.

[7] Warren, H.P., Cramer, R., Kidd, S. and Leichliter, J.S. (2018) State Requirements for Prenatal Syphilis Screening in the United States, 2016. Maternal and Child Health Journal, 22, 1227-1232. https://doi.org/10.1007/s10995-018-2592-0

[8] Park, I.U., Fakile, I.U., Chow, J.M., Gustafson, K.J., Jost, H., Schapiro, J.M., et al. (2019) Performance of Treponemal Tests for the Diagnosis of Syphilis. Clinical Infectious Diseases, 68, 913-918. https://doi.org/10.1093/cid/ciy558

[9] Shahrook, S., Mori, R., Ochirbat, T. and Gomi, H. (2014) Strategies of Testing for Syphilis during Pregnancy. Cochrane Database of Systematic Reviews. https://doi.org/10.1002/14651858.CD010385.pub2

[10] Negash, M., Wondmagegn, T. and Geremew, D. (2018) Comparison of RPR and ELISA with TPHA for the Diagnosis of Syphilis: Implication for Updating Syphilis Point-of-Care Tests in Ethiopia. Journal of Immunology Research, 2018, Article ID: 2978419. https://doi.org/10.1155/2018/2978419

[11] Workowski, K.A. and Berman, S. (2010) Centers for Disease Control and Prevention (CDC). Sexually Transmitted Diseases Guidelines. MMWR Recommendations and Reports, 59, 1-110.

[12] Trivedi, S., Taylor, M., Lamb, M.L. and Chou, D. (2020) Evaluating Coverage of Maternal Syphilis Screening and Treatment within Antenatal Care to Guide Service Improvements for Prevention of Congenital Syphilis in Countdown 2030 Countries. Journal of Global Health, 10, Article ID: 010504. https://doi.org/10.7189/jogh.10.010504

[13] Rowe, C.R., Newberry, D.M. and Jnah, A.J. (2018) Congenital Syphilis: A Discussion of Epidemiology, Diagnosis, Management, and Nurses' Role in Early Identification and Treatment. Advances in Neonatal Care, 18, 438-445. https://doi.org/10.1097/ANC.0000000000000534

[14] Amsalu, A., Ferede, G. and Assegu, D. (2018) High Seroprevalence of Syphilis Infection among Pregnant Women in Yiregalem Hospital Southern Ethiopia. BMC 
Infectious Diseases, 18, Article No. 109. https://doi.org/10.1186/s12879-018-2998-8

[15] Swartzendruber, A., Steiner, R.J., Alder, M.R., Kamb, M.L. and Newman, L.M. (2015) Introduction of Rapid Syphilis Testing in Antenatal Care: A Systematic Review of the Impact on HIV and Syphilis Testing Uptake and Coverage. International Journal of Gynecology \& Obstetrics, 130, 15-21.

https://doi.org/10.1016/j.ijgo.2015.04.008

[16] Nwosu, B.O., Eleje, G.U., Obi-Nwosu, A.L., Ahiarakwem, I.F., Akujobi, C.N., Egwuatu, C.C., et al. (2015) Is Routine Antenatal Venereal Disease Research Laboratory Test Still Justified? Nigerian Experience. International Journal of Women's Health, 7, 41-46. https://doi.org/10.2147/IJWH.S70442

[17] Phang Romeo, C.C., Martyn-St James, M., Hamilton, J., Marinho, D.S., Castro, R. and Harnan, S. (2018) Rapid Diagnostic Test for Antenatal Syphilis Screening in Low-Income and Middle-Income Countries: A Systematic Review and $\mathrm{Me}$ ta-Analysis. BMJ Open, 8, e018132. https://doi.org/10.1136/bmjopen-2017-018132

[18] Morshed, M.G. and Singh, A.E. (2015) Recent Trend in Serologic Diagnosis of Syphilis. Clinical and Vaccine Immunology, 22, 137-147.

https://doi.org/10.1128/CVI.00681-14

[19] Sena, A.C., White, B.L. and Sparling, A.F. (2010) Novel Treponema pallidum Serologic Tests: A Paradigm Shift in Syphilis Screening for the 21st Century. Clinical Infectious Diseases, 51, 700-708. https://doi.org/10.1086/655832

[20] Rac, M.W., Revell, P.A. and Eppes, C.S. (2017) Syphilis during Pregnancy: A Preventable Threat to Maternal-Fetal Health. American Journal of Obstetrics and Gynecology, 216, 352-363. https://doi.org/10.1016/j.ajog.2016.11.1052

[21] Bazzo, M.L., da Motta, L.R., Rudolf-Oliveira, R.C.M., Bigolin, A., Golfetto, L., Mesquita, F., et al. (2017) Evaluation of Seven Rapids Tests for Syphilis Available in Brazil Using Defibrinated Plasma Panels. Sexually Transmitted Infections, 93, 46-50. https://doi.org/10.1136/sextrans-2017-053177 